\title{
Finite deformation in ideal magnetohydrodynamics
}

\author{
D. MacTaggart
}

\author{
School of Engineering, Computing and Applied Mathematics, University of Abertay, Kydd Building, Dundee, DD1 1HG, UK \\ e-mail: D.MacTaggart@abertay.ac.uk
}

Received 7 January 2012 / Accepted 26 April 2012

\section{ABSTRACT}

\begin{abstract}
Aims. In this paper we investigate the finite deformation of magnetic fields that can enable one to find complex analytical magnetohydrostatic (MHS) equilibria. These can be used as input to non-linear simulations.

Methods. In order to find analytical equilibria, one normally has to consider simplifications or exploit a particular symmetry. Even with these measures, however, the desired equilibrium is often out of analytical reach. Here we describe a method that can work when traditional methods fail. It is based on the smooth deformation of simple magnetic fields into complex ones.

Results. Examples are given, to demonstrate the method, that are of practical importance in coronal physics. This technique will prove useful in setting up the initial conditions of non-linear magnetohydrodynamic simulations.
\end{abstract}

Key words. magnetic fields - magnetohydrodynamics (MHD) - methods: analytical

\section{Introduction}

The magnetohydrodynamic (MHD) equations are non-linear, making the search for analytical solutions difficult and interesting. Much progress has been made in the reduced problem of finding exact magnetohydrostatic (MHS) solutions. MHS equilibria are important for modelling purposes and as initial conditions for non-linear MHD simulations. The MHS equations are

$$
\begin{aligned}
\nabla p & =\boldsymbol{j} \times \boldsymbol{B}+\boldsymbol{G}, \\
\mu_{0} \boldsymbol{j} & =\nabla \times \boldsymbol{B}, \\
\nabla \cdot \boldsymbol{B} & =0,
\end{aligned}
$$

where $\boldsymbol{B}$ is the magnetic induction (commonly referred to as the magnetic field), $\boldsymbol{j}$ is the current density, $p$ is the plasma pressure, $\mu_{0}$ is the magnetic permeability of free space and $\boldsymbol{G}$ represents any possible body forces, e.g. gravity. There exists a plethora of methods for solving the above equations and their further simplifications. If the magnetic field has translational, rotational or helical symmetry, the MHS equations can be reduced to one single elliptic second order partial differential equation. This is known as a Grad-Shafranov equation (e.g. Biskamp 1997). For example, consider $\boldsymbol{G}=\mathbf{0}$ in Eq. (1) and assume that the invariant direction is the $y$-direction, i.e. $\partial / \partial y=0$. The magnetic field, satisfying Eq. (3), can be written as

$\boldsymbol{B}=\nabla \times\left(A \boldsymbol{e}_{y}\right)+B_{y} \boldsymbol{e}_{y}$

where $A$ is the flux function and $B_{y}$ is the $y$-component of the magnetic field. Both terms depend only on $x$ and $z$. After inserting this expression into Eqs. (1) and (2) and simplifying, the Grad-Shafranov equation is

$-\nabla^{2} A=\mu_{0} \frac{\mathrm{d} p}{\mathrm{~d} A}+B_{y} \frac{\mathrm{d} B_{y}}{\mathrm{~d} A}$.

The pressure $p$ and magnetic field component $B_{y}$ are free functions of $A$. These have to be chosen or specified by the application. For example, consider the application of coronal magnetic loops with footpoints anchored in a lower boundary (the photosphere). Since, in general, $B_{y}$ will not vanish, the footpoints of loops will be displaced in the $y$-direction. $B_{y}$ is related to this displacement via an integral equation. Only for the simplest fields can this be solved, in conjunction with the Grad-Shafranov equation, analytically. An account of this type of problem is given in Biskamp (1997).

For asymmetric fields or symmetric fields of suffiecient complexity, other ad hoc approaches are required to find MHS equilibria. One approach is to consider magnetic fields expressed by Euler potentials

$\boldsymbol{B}=\nabla \alpha \times \nabla y$,

where $\alpha=\alpha(x, y, z)$. The magnetic field has no $y$-component and the field lines are confined to planes of constant $y$. Equilibria with such magnetic fields are called laminated equilibria (Low 1982). Another approach that has been used successfully for force-free and MHS equilibria is to consider the current density in terms of Euler potentials (Low 1991, 1992; Neukirch 1997; Petrie \& Neukirch 2000).

Although there has been considerable success with the methods described above, they are not suitable for every application. In practice, MHS equilibria are often found numerically by deforming simple magnetic fields. These deformations usually take the form of flows imposed on the boundaries of a computational domain. Once the deformation is complete, the magnetic field is left to relax into equilibrium. Based on this technique, we shall now present a method for deforming simple magnetic fields into complex ones, analytically. This then allows for an analytical MHS equilibrium to be found. The method can free the computing time and resources previously tied up in creating the equilibrium by boundary driving and relaxation.

The rest of the paper is laid out as follows: Sect. 2 introduces the deformation theory. Section 3 presents some illustrative examples of the theory with applications in coronal physics. The paper concludes with a summary and discussion in Sect. 4. 


\section{Deformation theory}

\subsection{Introduction}

Consider a continuous plasma (or fluid) that occupies a connected open subset of a three dimensional Euclidean point space. We will refer to such a subset as a configuration of the plasma. Given a magnetic field, we can choose the plasma to be in an arbitrary configuration, known as a reference configuration. This can be deformed quasi-statically to a new configuration, known as the current configuration. Normally in ideal MHD, this approach takes the form of a Cauchy solution, where a velocity field $\boldsymbol{u}$ is prescribed and the deformed magnetic field is found from the induction equation

$$
\frac{\partial \boldsymbol{B}}{\partial t}=\nabla \times(\boldsymbol{u} \times \boldsymbol{B})
$$

If, however, we are only interested in MHS equilibria, we can avoid prescribing a velocity to deform the magnetic field and, hence, ignore the induction equation. This is done by prescribing the deformation in terms of the coordinate systems of the reference and current configurations - a technique more common to solid mechanics (Ogden 1997). In order to deform magnetic fields smoothly in this way, several conditions must be met. These are described in the rest of this section.

\subsection{Deformation gradient}

Let a typical point of the plasma be labelled by its position vector $\boldsymbol{X}$ in the reference configuration $C_{\mathrm{r}}$ and $\boldsymbol{x}$ in the current configuration $C_{\mathrm{t}}$. We suppose the plasma is deformed smoothly ${ }^{1}$ from one configuration to the other via the mapping

$x=x(X)$.

We ignore time dependence here. The vector field $x$ describes the deformation of the plasma and is defined for $\boldsymbol{X} \in C_{\mathrm{r}}$. The deformation gradient tensor $\mathbf{F}$ relative to $C_{\mathrm{r}}$ is defined by

$\mathbf{F}=\operatorname{Grad} \boldsymbol{x}=\frac{\partial \boldsymbol{x}}{\partial \boldsymbol{X}}$

Grad denotes the gradient in the reference configuration $C_{\mathrm{r}}$. For a fixed time, line elements $\mathrm{d} \boldsymbol{X}$ and $\mathrm{d} \boldsymbol{x}$ are connected by

$\mathrm{d} \boldsymbol{x}=\frac{\partial \boldsymbol{x}}{\partial \boldsymbol{X}} \mathrm{d} \boldsymbol{X}$

Since a line element cannot disappear, we must have $\operatorname{det} \mathbf{F} \neq 0$. If we define volume elements to be positive, it follows (Ogden 1997) that

$J=\operatorname{det} \mathbf{F}>0$.

In $C_{\mathrm{r}}$ a magnetic field must satisfy

Div $\boldsymbol{B}_{\mathrm{r}}=0$,

where $\boldsymbol{B}_{\mathrm{r}}$ is the magnetic field in $C_{\mathrm{r}}$ and Div is the divergence operator in $C_{\mathrm{r}}$. When the plasma is deformed with deformation gradient $\mathbf{F}$, we denote the corresponding deformed field in $C_{\mathrm{t}}$ by $\boldsymbol{B}_{\mathrm{t}}$. In $C_{\mathrm{t}}$, the magnetic field must also be divergence-free. We have

$\operatorname{Div} \boldsymbol{B}_{\mathrm{r}}=J \operatorname{div}\left(J^{-1} \mathbf{F} \boldsymbol{B}_{\mathrm{r}}\right)=0$,

\footnotetext{
${ }^{1}$ In this paper, the mapping is a diffeomorphism.
}

where div is the divergence operator in $C_{\mathrm{t}}$. A derivation of Eq. (6) is given in the appendix. From the expression for the divergence in (6), it follows that

$\boldsymbol{B}_{\mathrm{t}}=J^{-1} \mathbf{F} \boldsymbol{B}_{\mathrm{r}}$.

This is the new deformed magnetic field in $C_{\mathrm{t}}$.

Unlike the magnetic field, there is no simple connection relating the reference and current current densities. In the reference configuration we have

$\operatorname{Curl} \boldsymbol{B}_{\mathrm{r}}=\boldsymbol{j}_{\mathrm{r}}$,

and, similarly, in the current configuration,

$\operatorname{curl} \boldsymbol{B}_{\mathrm{t}}=\boldsymbol{j}_{\mathrm{t}}$.

By making use of the connection between the Curl and curl operators (see the appendix), the deformed current density can be written in terms of the reference magnetic field and the deformation

$\boldsymbol{j}_{\mathrm{t}}=J^{-1} \mathbf{F C u r l}\left(J^{-1} \mathbf{c} \boldsymbol{B}_{\mathrm{r}}\right)$,

where $\mathbf{c}=\mathbf{F}^{\mathrm{T}} \mathbf{F}$ is the right Cauchy-Green deformation tensor.

For the rest of the paper, we shall only be concerned with the deformation of the magnetic field. We do not consider any constraints on the current density, such as ensuring force-free conditions. Any equilibria are determined after the deformation.

\section{Examples}

In this section we shall illustrate the theory with some simple examples. First, we shall demonstrate how to construct a deformation gradient tensor and apply it to deforming a magnetic null point. This is followed by two further applications that are of practical importance in coronal physics.

\subsection{Null point deformation}

To construct a deformation gradient tensor, one must choose a deformation (e.g. a shear, a twist, etc.) and find a suitable mapping from the reference to the current configuration that describes this deformation. We shall illustrate this here by applying a shear to a magnetic null point.

To put this application into context, null points are of interest as regions where magnetic reconnection is likely to occur. There have been many simulations of stressed null points (see Galsgaard \& Pontin 2011, and references therein). In these simulations, the null points are sheared by imposing flows on the boundaries of the computational domain. This allows one to study current build-up due to shearing or, if the driving is ceased, the subsequent relaxation. Using the deformation theory introduced in the previous section, one could shear a null point to produce the same configuration as boundary shearing would in a simultation. If one was interested in studying the relaxation of a sheared null point, the application of this method would remove the need for initial numerical shearing.

To begin, let us consider a simple, linear, potential null point defined by

$B_{X}=B_{0} X, \quad B_{Y}=B_{0} Y, \quad B_{Z}=-2 B_{0} Z$,

where $B_{0}$ is a constant. Note that this is a local representation of the magnetic field and is only valid close to the null point. Here we will consider the domain $(X, Y, Z) \in[-l, l]^{3}$, where $l$ is 

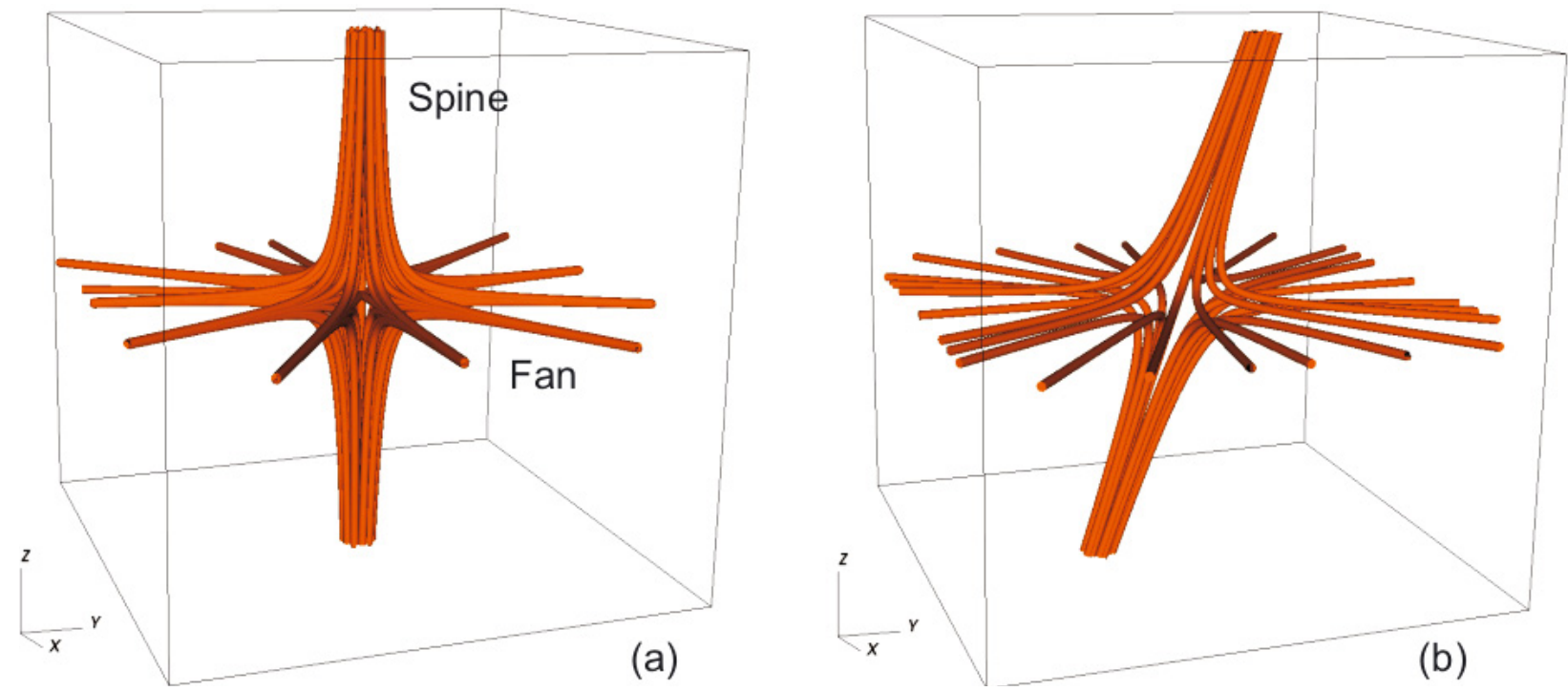

Fig. 1. A magnetic null point before and after shearing. a) shows the undeformed (reference configuration) potential null point. The spine and the fan are highlighted. b) shows the effect of the shearing deformation (current configuration).

a suitably small distance from the null point. The position variables $X, Y$ and $Z$ are written in upper-case. We use the convention of upper-case for quantities in the reference configuration and lower-case for those in the current configuration. An image of the shape of the field lines about the undeformed null point is displayed in Fig. 1a.

The null point can be described as having a spine and a fan (as shown in Fig. 1a). Suppose we want to shear the null point by pushing the two ends of the spine in opposite directions (as in Galsgaard \& Pontin 2011). By "ends", we refer to where the spine meets the boundary of the computational domain. To proceed, we need to find a mapping that will give us the profile we want and satisfy Eq. (5). For shearing the spine, consider a mapping of the form

$x=X, \quad y=Y+g Z, \quad z=Z$,

where $g$ is an arbirtary function. Here, the only change appears in the expression for the $y$ coordinate - the direction of shearing. As we are shearing in the $(Y, Z)$-plane, $y$ is equal to $Y$ plus a multiple of $Z$. For this illustrative example, we shall take $g=\gamma$, a constant.

We now have a mapping that will shear the two ends of the spine in opposite directions. To construct the deformation gradient tensor, we substitute the mapping into Eq. (4) to get, in matrix form,

$[\mathbf{F}]=\left(\begin{array}{lll}1 & 0 & 0 \\ 0 & 1 & \gamma \\ 0 & 0 & 1\end{array}\right)$.

It is clear that $J>0$. The magnetic field vector for the linear null point is now multiplied by the deformation gradient tensor. At this point, all the variables are with respect to the reference configuration $C_{\mathrm{r}}$. To switch to the current configuration $C_{\mathrm{t}}$, one applies the mapping (7) to obtain

$B_{x}=B_{0} x, \quad B_{y}=B_{0}(y-\gamma z), \quad B_{z}=-2 B_{0} z$.

Figure 1b shows how the applied shearing deforms the null point.

This example demonstrates how one can construct and apply a deformation gradient tensor. We shall now consider two further examples from coronal physics, where we shall use the deformation theory to find full MHS equilibria.

\subsection{Sheared arcade}

Satellites have revealed the solar atmosphere to be filled with magnetic loops. Magnetic arcades play an important role in a variety of solar atmospheric phenomena. Long-lasting (relative to some characteristic time such as the photospheric Alfvén time) arcades can be modelled as equilibria. A classic problem in coronal physics is the sheared arcade equilibrium. The "standard solution" is to assume a linear force-free magnetic field. The Grad-Shafranov equation combined with an equation relating the footpoint displacement with $B_{y}$ is then solved. A full MHS solution is possible by deforming an unsheared potential arcade. First, consider a simple potential arcade defined by

$B_{X}=B_{0} \cos \left(\frac{\pi X}{l}\right) \exp \left(-\frac{\pi Z}{l}\right)$

$B_{Y}=0$,

$B_{Z}=-B_{0} \sin \left(\frac{\pi X}{l}\right) \exp \left(-\frac{\pi Z}{l}\right)$,

where $B_{0}$ and $l$ are constants. The base, $Z=0$, represents the photosphere and $X \in[-l, l]$. Again, upper-case is used for $X, Y$ and $Z$ here to signify that the variables are in the reference configuration $C_{\mathrm{r}}$ and lower-case will be used for the current configuration $C_{\mathrm{t}}$. Similar to the previous example, we will now perform a simple shear in the $(X, Y)$-plane to deform this potential arcade. Consider a deformation of the form

$x=X, \quad y=Y+\gamma X, \quad z=Z$,

where $\gamma$ is a dimensionless constant. Let $\left\{\boldsymbol{E}_{X}, \boldsymbol{E}_{Y}, \boldsymbol{E}_{Z}\right\}$ and $\left\{\boldsymbol{e}_{x}, \boldsymbol{e}_{y}, \boldsymbol{e}_{z}\right\}$ be Cartesian bases for $C_{\mathrm{r}}$ and $C_{\mathrm{t}}$, respectively. The deformation gradient is given by

$\mathbf{F}=\mathbf{I}+\gamma \boldsymbol{e}_{y} \otimes \boldsymbol{E}_{X}$

where $\mathbf{I}$ is the identity tensor. The matrix representation of the deformation gradient is

$[\mathbf{F}]=\left(\begin{array}{lll}1 & 0 & 0 \\ \gamma & 1 & 0 \\ 0 & 0 & 1\end{array}\right)$. 
It follows that, for simple shear, $J=1$. This satisfies (5) and, mechanically, represents an isochoric (or volume preserving) deformation. Since $\mathbf{F}$ is a constant, this is known as a homogeneous deformation. The deformed magnetic field is, therefore, given by

$$
\begin{aligned}
& B_{x}=B_{0} \cos \left(\frac{\pi x}{l}\right) \exp \left(-\frac{\pi z}{l}\right), \\
& B_{y}=\gamma B_{0} \cos \left(\frac{\pi x}{l}\right) \exp \left(-\frac{\pi z}{l}\right), \\
& B_{z}=-B_{0} \sin \left(\frac{\pi x}{l}\right) \exp \left(-\frac{\pi z}{l}\right) .
\end{aligned}
$$

This magnetic field is not force-free (assuming $\gamma \neq 0$ ). The Lorentz force is irrotational, i.e.

$\operatorname{curl}(\boldsymbol{j} \times \boldsymbol{B})=\mathbf{0}$

This means a MHS equilibrium can be found by solving, directly, for $p$ in Eq. (1) with $\boldsymbol{G}=\mathbf{0}$. The result, after some simple algebra, is

$p=p_{0}-\frac{\gamma^{2} B_{0}^{2}}{2 \mu_{0}} \cos ^{2}\left(\frac{\pi x}{l}\right) \exp \left(-\frac{2 \pi z}{l}\right)$,

where $p_{0}$ is a constant that satisfies

$p_{0}>\frac{\gamma^{2} B_{0}^{2}}{2 \mu_{0}}$

to ensure a positive pressure.

\subsection{Flux braiding}

The twisting and entangling of coronal magnetic field lines by photospheric footpoint motions is one of the prime candidates for coronal heating. Braiding causes a build up of magnetic energy and the formation of strong concentrations of current density, allowing reconnection to take place. There have been several numerical studies where, initially simple, magnetic fields are deformed by imposing shear/rotational flows on the boundaries (e.g. Craig et al. 1986; Longbottom et al. 1998; Galsgaard 2002; De Moortel \& Galsgaard 2006). These motions can produce regions of twisted current concentrations. In the case of Longbottom et al. (1998), they solve the force-free equations and find equilibria with twisted current concentrations. Attempts to find an analytical expression for twisted current concentrations, based on the Grad-Shafranov equation, have proved to be difficult (Bowness 2011). Here we shall present an analytical MHS equilibrium containing a twisted current concentration, based on deformation theory. Consider a domain $(X, Y, Z) \in$ $[-l, l]^{3}$ and a uniform magnetic field given by

$B_{X}=0, \quad B_{Y}=0, \quad B_{Z}=B_{0}$,

where $B_{0}$ is a constant. Since braiding is caused by shearing and twisting motions, consider the shearing deformation

$x=X, \quad y=Y+\lambda \sin \left(\frac{\pi X}{l}\right) Z, \quad z=Z$,

where $\lambda$ is a dimensionless constant. The corresponding deformation gradient is given by

$\mathbf{F}=\mathbf{I}+\frac{\lambda \pi}{l} \cos \left(\frac{\pi X}{l}\right) Z \boldsymbol{e}_{y} \otimes \boldsymbol{E}_{X}+\lambda \sin \left(\frac{\pi X}{l}\right) \boldsymbol{e}_{y} \otimes \boldsymbol{E}_{Z}$.

A97, page 4 of 6
This inhomogeneous deformation is isochoric $(J=1)$ and the deformed magnetic field in $C_{\mathrm{t}}$ is

$B_{x}=0, \quad B_{y}=B_{0} \lambda \sin \left(\frac{\pi x}{l}\right), \quad B_{z}=B_{0}$.

To produce a twisted current concentration, we shall now apply a torsional deformation to the magnetic field in (10). There is a new current configuration $C_{\mathrm{t}}^{\prime}$ with $C_{\mathrm{t}}$ now acting as the reference configuration. Consider a deformation in which each crosssection remains in its original plane but is rotated through an angle $\tau z$ about the $z$-axis. $\tau$ is the constant twist per unit length. In the $(x, y)$-plane, the coordinates can be written in polar form

$x=r \cos \theta, \quad y=r \sin \theta$.

The torsional deformation can now be written as

$x^{\prime}=r \cos (\tau z+\theta), \quad y^{\prime}=r \sin (\tau z+\theta), \quad z^{\prime}=z$.

Given that $\left\{\boldsymbol{e}_{x}^{\prime}, \boldsymbol{e}_{y}^{\prime}, \boldsymbol{e}_{z}^{\prime}\right\}$ is the Cartesian basis of $C_{\mathrm{t}}^{\prime}$, the corresponding deformation gradient is, in matrix form,

$\left[\mathbf{F}^{\prime}\right]=\left[\frac{\partial \boldsymbol{x}^{\prime}}{\partial \boldsymbol{x}}\right]=\left(\begin{array}{ccc}\cos (\tau z) & -\sin (\tau z) & -\tau(\sin (\tau z) x+\cos (\tau z) y) \\ \sin (\tau z) & \cos (\tau z) & \tau(\cos (\tau z) x-\sin (\tau z) y) \\ 0 & 0 & 1\end{array}\right)$.

Again, $J=1$. The deformed magnetic field is given by

$B_{x}=-B_{0} \lambda \sin (\tau z) \sin \left(\frac{\pi}{l}(\cos (\tau z) x+\sin (\tau z) y)\right)-B_{0} \tau y$,

$B_{y}=B_{0} \lambda \cos (\tau z) \sin \left(\frac{\pi}{l}(\cos (\tau z) x+\sin (\tau z) y)\right)+B_{0} \tau x$,

$B_{z}=B_{0}$.

Here we have dropped the primes for simplicity. All further variables and operators are associated with the current configuration, so there should be no confusion as to what they represent. The corresponding components of the current density are given by

$$
\begin{aligned}
j_{x}= & \frac{B_{0} \lambda \tau}{\mu_{0}}\left[\sin (\tau z) \sin \left(\frac{\pi}{l}(\cos (\tau z) x+\sin (\tau z) y)\right)\right. \\
& -\frac{\pi}{l} \cos (\tau z)(\cos (\tau z) y-\sin (\tau z) x) \\
& \left.\times \cos \left(\frac{\pi}{l}(\cos (\tau z) x+\sin (\tau z) y)\right)\right], \\
j_{y}= & -\frac{B_{0} \lambda \tau}{\mu_{0}}\left[\cos (\tau z) \sin \left(\frac{\pi}{l}(\cos (\tau z) x+\sin (\tau z) y)\right)\right. \\
& +\frac{\pi}{l} \sin (\tau z)(\cos (\tau z) y-\sin (\tau z) x) \\
& \left.\times \cos \left(\frac{\pi}{l}(\cos (\tau z) x+\sin (\tau z) y)\right)\right], \\
j_{z}= & \frac{B_{0} \lambda \pi}{\mu_{0} l} \cos \left(\frac{\pi}{l}(\cos (\tau z) x+\sin (\tau z) y)\right)+\frac{2 B_{0} \tau}{\mu_{0}} .
\end{aligned}
$$

Figure 2 displays the field line and current concentration geometries for the deformed magnetic field. In Fig. 2a, the effects of the sine shear profile and the twist can be seen clearly. The isosurface of $|\boldsymbol{j}|$ in Fig. $2 b$ is highly reminiscient of that from the numerical study by Longbottom et al. (1998).

Unlike the sheared arcade, the Lorentz force in this example is not irrotational. Hence, we cannot solve Eq. (1) with $\boldsymbol{G}=\mathbf{0}$ directly for $p$. To find the plasma pressure for MHS balance, we need to make the RHS of Eq. (1) irrotational. To do this we must consider $\boldsymbol{G} \neq \mathbf{0}$. A, physically sensible, choice for $\boldsymbol{G}$ is

$\boldsymbol{G}=-\rho(x, y, z) g \boldsymbol{e}_{z}$, 


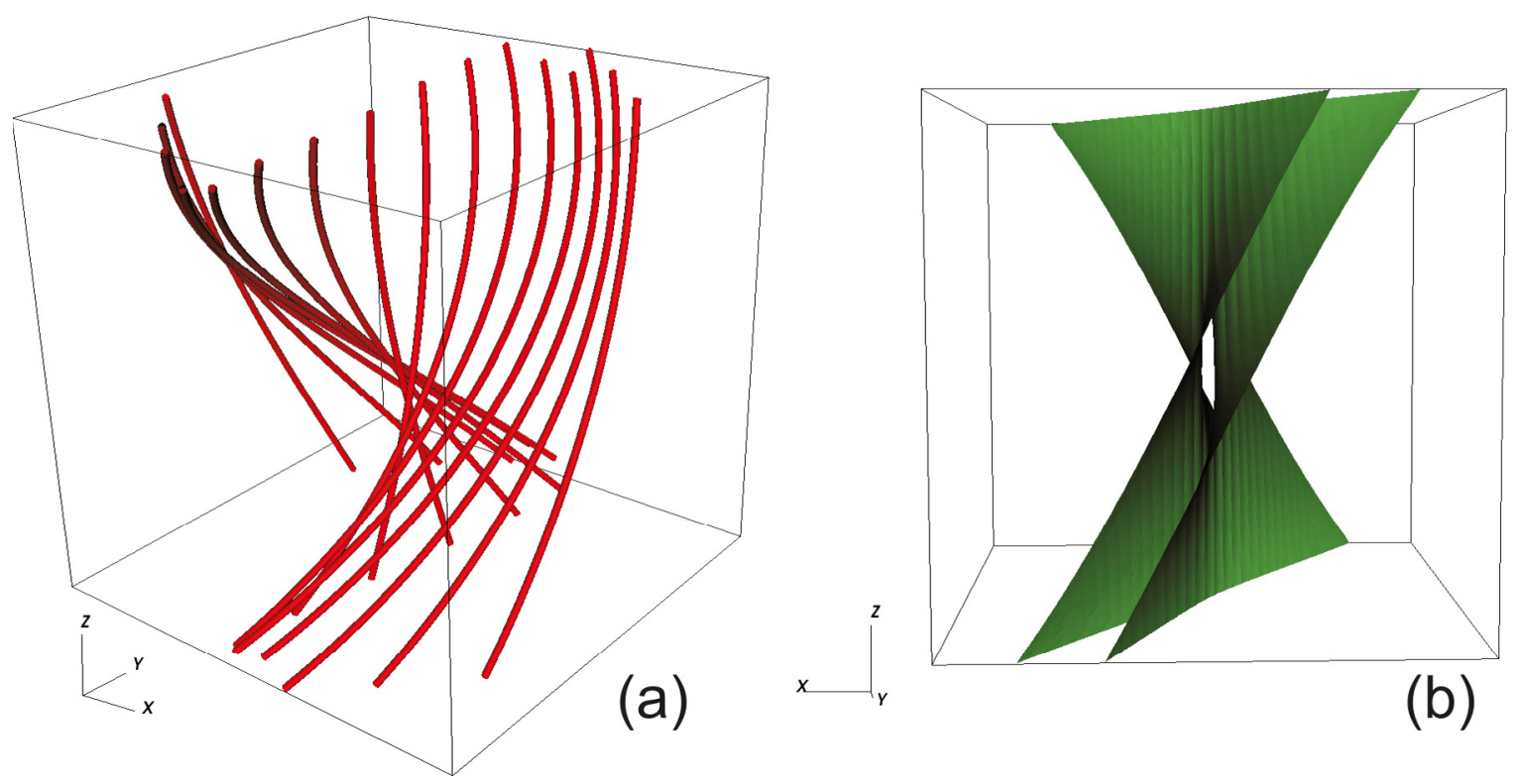

Fig. 2. The magnetic field after the shearing and torsional deformations. a) shows some magnetic field lines. b) displays an isosurface of $|j|$ at $90 \%$ of the maximum value.

where $\rho$ is the density and $g$ is the gravitational constant. Solving

$\operatorname{curl}\left(\boldsymbol{j} \times \boldsymbol{B}-\rho g \boldsymbol{e}_{z}\right)=\mathbf{0}$,

for the density gives

$$
\begin{aligned}
\rho= & \rho_{0}+\frac{2 B_{0}^{2} \lambda \tau^{2}}{g \mu_{0}} \sin \left(\frac{\pi}{l}(\cos (\tau z) x+\sin (\tau z) y)\right) \\
& \times(\cos (\tau z) y-\sin (\tau z) x) .
\end{aligned}
$$

$\rho_{0}$ is a function of $z$ that must be chosen to ensure the density is positive within the domain. This will, in general, depend on $l$. Now that the RHS of Eq. (1) is irrotational, we can integrate to find the pressure. The result is

$$
\begin{aligned}
p= & p_{0}+\frac{B_{0}^{2}}{\mu_{0}}\left[\frac{2 l \lambda \tau}{\pi} \cos \left(\frac{\pi}{l}(\cos (\tau z) x+\sin (\tau z) y)\right)\right. \\
& -\frac{\lambda^{2}}{2} \sin ^{2}\left(\frac{\pi}{l}(\cos (\tau z) x+\sin (\tau z) y)\right) \\
& -\lambda \tau \sin \left(\frac{\pi}{l}(\cos (\tau z) x+\sin (\tau z) y)\right)(\cos (\tau z) x+\sin (\tau z) y) \\
& \left.-\tau^{2}\left(x^{2}+y^{2}\right)\right]-\rho_{0} g z
\end{aligned}
$$

$p_{0}$ is a constant that must be chosen to ensure a positive pressure within the domain.

\section{Summary and discussion}

In this paper, we have added to the arsenal of methods for finding analytical MHS equilibria. Inspired by the numerical technique of imposing flows on a simple magnetic field and then leaving the system to relax, this new method imposes quasi-static deformations that can can be treated analytically. Once the magnetic field is suitably deformed, the density can be found by making the RHS of Eq. (1) irrotational. The scalar potential of this is then the pressure. The advantage of this method over traditional ones is that analytical expressions for complex magnetic fields can be found with relative ease. Three examples of this are given. The first demonstrates how to construct and apply the deformation gradient tensor. In this example, it is applied to shearing a null point.

The second is a classic problem in coronal physics - the sheared arcade. Normally, an analytical approach is restricted to considering a linear force-free magnetic field. With this method, however, a full MHS solution is found by deforming an unsheared potential arcade.

The third example gives an expression for a twisted current concentration. Bowness (2011) attempt to produce such a configuration using a Grad-Shafranov equation. This does not give the desired result, however, and they produce their initial condition by deforming the field numerically.

It is not indended that this method will replace previous ones but complement them. Its success, as mentioned above, lies in deriving analytical expressions for complex magnetic fields and currents with relative ease. The method does have some drawbacks, however. One is that for the identity in (6) to hold, the mapping must be suitably well behaved. This means that fields cannot be deformed with impunity. Another drawback, as illustrated by the examples described in this paper, is that the density and pressure often rely on boundary values to ensure they are positive. i.e. the equilibria are local, not global, solutions. This drawback is also associated with more traditional methods (e.g. Grad-Shafranov) of finding analytical equilibria.

In practice, we believe that the advantages of the method outweigh the disadvantages. Although the number of deformations is limited, it is a large number and allows for the derivation of a vast range of highly complex magnetic fields from simple ones. By the examples given, we have demonstrated that the method can succeed where others fail. It is likely that the method will be used most in finding magnetic fields for the initial conditions of non-linear simulations. This will save a large amount of memory and computing time previously used in calculating the initial magnetic fields by the imposition of driving velocities on the boundaries.

\section{Appendix A: Derivation of identities}

Here, we sketch the proofs of the identity relations required for the deformation of the magnetic field and the current density. 


\section{A.1. The divergence identity}

By Gauss' theorem, the integral of the divergence of $\boldsymbol{B}_{0} \in C$ over the volume of $C_{\mathrm{r}}$ can be written as a surface integral

$\int_{C_{\mathrm{r}}} \operatorname{Div} \boldsymbol{B}_{0} \mathrm{~d} V=\int_{\partial C_{\mathrm{r}}} \boldsymbol{B}_{0} \cdot \boldsymbol{N} \mathrm{d} S$,

where $\partial C_{\mathrm{r}}$ is the boundary of $C_{\mathrm{r}} . V$ represents volume and $S$, surface area. Nanson's formula relates the area differentials of the reference and current frames

$\boldsymbol{N} \mathrm{d} S=J^{-1} \mathbf{F}^{\mathrm{T}} \boldsymbol{n} \mathrm{d} s$.

As in the main body of the text, we use upper-case for the reference configuration and lower-case for the current configuration. It follows that the surface integral in (A.1), with respect to $C_{\mathrm{t}}$ is

$\int_{\partial C_{\mathrm{t}}} \boldsymbol{B}_{0} \cdot\left(J^{-1} \mathbf{F}^{\mathrm{T}} \boldsymbol{n}\right) \mathrm{d} s=\int_{\partial C_{\mathrm{t}}} J^{-1} \mathbf{F} \boldsymbol{B}_{0} \cdot \boldsymbol{n} \mathrm{d} s$.

By Gauss' theorem,

$\int_{\partial C_{\mathrm{t}}} J^{-1} \mathbf{F} \boldsymbol{B}_{0} \cdot \boldsymbol{n} \mathrm{d} s=\int_{\mathcal{C}_{\mathrm{t}}} \operatorname{div}\left(J^{-1} \mathbf{F} \boldsymbol{B}_{0}\right) \mathrm{d} v$.

To move back to the reference frame, the volume differentials are related by

$\mathrm{d} v=J \mathrm{~d} V$.

Hence, it follows that

$\int_{C_{\mathrm{r}}} \operatorname{Div} \boldsymbol{B}_{0} \mathrm{~d} V=\int_{C_{\mathrm{r}}} J \operatorname{div}\left(J^{-1} \mathbf{F} \boldsymbol{B}_{0}\right) \mathrm{d} V$

and this completes the proof.

\section{A.2. The curl identity}

An application of Stoke's theorem for $\boldsymbol{B}_{\mathrm{t}} \in C_{\mathrm{t}}$ gives

$\int_{\partial C_{\mathrm{t}}} \operatorname{curl} \boldsymbol{B}_{\mathrm{t}} \cdot \boldsymbol{n} \mathrm{d} s=\int_{\Gamma_{\mathrm{t}}} \boldsymbol{B}_{\mathrm{t}} \cdot \boldsymbol{n} \mathrm{d} x$

where $\Gamma_{t}$ is the bounding curve. By repositioning the deformation gradient and applying Stoke's theorem, one can find

$\int_{\Gamma_{\mathrm{r}}} \boldsymbol{B}_{\mathrm{t}} \cdot \mathbf{F} \boldsymbol{N} \mathrm{d} X=\int_{\partial C_{\mathrm{r}}} \operatorname{Curl}\left(\mathbf{F}^{T} \boldsymbol{B}_{\mathrm{t}}\right) \cdot \boldsymbol{N} \mathrm{d} S$.

Using Nanson's fomula,

$\int_{\partial \mathcal{C}_{\mathrm{t}}} \operatorname{Curl}\left(\mathbf{F}^{T} \boldsymbol{B}_{\mathrm{t}}\right) J^{-1} \mathbf{F}^{T} \boldsymbol{n} \mathrm{d} s=\int_{\partial \mathcal{C}_{\mathrm{t}}} J^{-1} \mathbf{F} \operatorname{Curl}\left(\mathbf{F}^{T} \boldsymbol{B}_{\mathrm{t}}\right) \cdot \boldsymbol{n} \mathrm{d} s$.

To connect $\boldsymbol{j}_{\mathrm{t}}$ with $\boldsymbol{B}_{\mathrm{r}}$, insert $\boldsymbol{B}_{\mathrm{t}}=J^{-1} \mathbf{F} \boldsymbol{B}_{\mathrm{r}}$ into the last integral.

\section{References}

Biskamp, D. 1997, Nonlinear Magnetohydrodynamics (Cambridge University Press)

Bowness, R. 2011, Ph.D. Thesis, University of St Andrews

Craig, I. J. D., \& Sneyd, A. D. 1986, ApJ, 311, 451

De Moortel, I., \& Galsgaard, K. 2006, A\&A, 451, 1101

Galsgaard, K. 2002, SOLMAG 2002, Proc. Magnetic Coupling of the Solar Atmosphere Euroconference, 505, 269

Galsgaard, K., \& Pontin, D. 2011, A\&A, 534, A2

Longbottom, A. W., Rickard, G. J., Craig, I. J. D., \& Sneyd, A. D. 1998, ApJ, 500,471

Low, B. C. 1982, ApJ, 263, 952

Low, B. C. 1991, ApJ, 370, 427

Low, B. C. 1992, ApJ, 399, 300

Neukirch, T. 1997, A\&A, 325, 847

Ogden, R. W. 1997, Non-linear Elastic Deformations (Dover Publications)

Petrie, G. J. D., \& Neukirch, T. 2000, A\&A, 356, 735 